\title{
Cyclin E expression and proliferation in breast cancer
}

\author{
Niels H. Nielsen ${ }^{\mathrm{a}, \mathrm{b}}$, Conny Arnerlöv ${ }^{\mathrm{c}}$, Stefan Cajander ${ }^{\mathrm{a}}$ and Göran Landberg a,* \\ ${ }^{a}$ Department of Pathology, Umeå University, S-901 87 Umeå, Sweden \\ ${ }^{\mathrm{b}}$ Department of Oncology, Umeå University, S-901 87 Umeå, Sweden \\ ${ }^{\mathrm{c}}$ Department of Surgery, Umeå University, S-901 87 Umeå, Sweden
}

Received 14 April 1998

Revised 16 November 1998

Accepted 2 December 1998

\begin{abstract}
Cyclin E is a part of the cell cycle machinery and aberrantly expressed in several malignancies including breast cancer. Since cyclin E is cell cycle specifically expressed, we wanted to examine the relation between proliferation and expression of cyclin E with special attention to tumours with overexpression of the protein. Seventy-four breast tumours were analysed for the expression of cyclin $\mathrm{E}$ by immunohistochemistry and Western blotting and related to the growth fraction determined by $\mathrm{Ki}$ 67. Significant correlations were obtained between the growth fraction, the percentage of cyclin E positive cells, the intensity of cyclin E and total amount of cyclin E determined by Western blotting. The majority of the tumours had less cyclin E than Ki-67 positive cells indicating a conserved cell cycle specific expression of the protein which further was supported by flow cytometric analysis of breast cancer cell lines. The cell cycle specificity of cyclin E was found even in tumours with inactivated retinoblastoma protein $(\mathrm{pRB})$ demonstrating the existence of a $\mathrm{pRB}$ independent regulation of cyclin $\mathrm{E}$. A fraction of the tumours had considerably elevated cyclin E levels that were not in relation to the proliferative activity as observed for the other tumours. These tumours were in general highly proliferative and considered to overexpress cyclin E. Patients with tumours of high proliferative activity, high total cyclin $E$ levels or disproportionally elevated cyclin E expressions in relation to proliferation had significantly increased risk of death in breast cancer, whereas the intensity of the immunohistochemical cyclin E staining did not affect the survival.

Keywords: Cyclin E, proliferation, cell cycle, breast cancer, Ki-67, prognosis
\end{abstract}

\section{Introduction}

Cyclins are units in the important cell cycle regulatory complexes consisting of cyclin dependent kinases (CDKs), CDK-inhibitors and various cyclins. By their periodic expression, cyclins activate the CDKs at specific points in the cell cycle thereby causing phosphorylation of substrates necessary for cell cycle progression. Cyclins D1 and E are responsible for activation of CDKs during the G1 phase and rate limiting for the G1-S transition [2,28]. Cyclin E that associate with CDK2 and peaks in late G1 after cyclin D1 coinciding with the commitment of a cell to a new round of replication, is a potential restriction point protein essential for control of DNA replication initiation.

Because of the critical function of cyclins D1 and E in the cell cycle these proteins are potential targets in tumourigenesis. Cyclin D1 has been proposed to be a proto-oncogene because of its ability to transform fibroblasts either alone or with various established oncogenes $[13,20]$, to induce tumours in

${ }^{*}$ Corresponding author: Göran Landberg, Department of Pathology, Umeå University, S-901 87 Umeå, Sweden. Tel.: (46) 90 7852873; Fax: (46) 90 7852829; E-mail: goran.landberg.us@ vll.se. 
transgenic mice overexpressing cyclin D [31] and the finding of chromosomal rearrangements encompassing the cyclin D1 gene on 11q13 in diverse types of cancers [5,9,30]. For cyclin E there have been reports indicating that the protein is overexpressed in several malignancies and cell lines $[5,15,16]$ and recently we showed that approximately $25 \%$ of primary breast cancers expressed elevated levels of cyclin E determined by Western blotting correlating strongly to poor prognosis [25]. Amplifications of the cyclin $\mathrm{E}$ gene have been reported in digestive tract and ovarian cancers $[1,18]$ but seem to be rare in breast cancer [6]. Further studies are needed to define the mechanisms responsible for cyclin E overexpression in tumours and the consequences for the cell cycle machinery before the role of deregulated cyclin $\mathrm{E}$ in breast cancer pathogenesis can be established.

In an immunohistochemical study it was recently proposed that cyclin E could be used as a proliferation marker in breast tumours due to its cell cycle specific expression [8]. A strong correlation between the fraction of cyclin E and Ki-67 positive cells was demonstrated with some exceptions which were proposed to represent cyclin E overexpressing tumours. Other studies have suggested that tumours with deregulated cyclin E loses the cell cycle specific expression of cyclin E and express constant levels of the protein [17]. These conclusions were based mainly on experiments with a limited number of cell lines and we therefore wanted to investigate the cell cycle specific expression of cyclin $\mathrm{E}$ in a larger material of breast tumours. Furthermore, we wanted to analyse the relationship between proliferation and cyclin $\mathrm{E}$ protein expression in breast cancer in order to clarify the proliferative activity of cyclin E overexpressing tumours. In summary, we found a close relationship between the percentage of cyclin E positive tumour cells and the growth fraction even though some tumours expressed high levels of cyclin $\mathrm{E}$ that were not in relation to the proliferative activity, suggesting that cyclin $\mathrm{E}$ was truly overexpressed in a fraction of the tumours.

\section{Material and methods}

\subsection{Patient data}

The patients in this study have previously been described [25] and consisted of women with operable primary breast cancer who attended Umeå University Hospital during 1988-1991. No patient received antitumoural therapy prior to surgery. Of the 74 tumours included in the study were 67 ductal carcinomas, two mucinous carcinomas, two lobular carcinomas and three medullary carcinomas.

\subsection{Flow cytometry of cell lines}

Breast cancer cell lines obtained from American Type Culture Collection (ATCC) were all grown in RPMI 1640 medium supplemented with $10 \%$ foetal calf serum and antibiotics. Cell lines were harvested with trypsin, fixed in methanol for $10 \mathrm{~min}$ and then incubated with monoclonal mouse anti-cyclin $\mathrm{E}$ antibodies (HE 12, Santa Cruz Inc., USA) diluted 1:50 for $30 \mathrm{~min}$ at $+4^{\circ} \mathrm{C}$. After washes in PBS cells were incubated with FITC-conjugated rabbit anti-mouse antibodies and after additional washes resuspended in a DNA staining solution containing propidium iodide and RNAse before flow cytometric analysis using a FACScan (Becton-Dickinson Inc., USA). Acquisition and analysis were performed with the Lysys 2 program (Becton-Dickinson). Debris and doublets were discriminated using gates on FSCSSC and FL2 width and FL2 area. 


\subsection{Immunohistochemistry}

Breast cancer specimens were fixed in buffered formalin $(\mathrm{pH} 7.0)$ and embedded in paraffin according to the clinical routine at the time of surgery. The fixation time in formalin varied slightly between different samples but were in general between 15-24 hrs. Three $\mu \mathrm{m}$ paraffin sections were prepared from paraffin embedded blocks, dried and then deparaffinized in xylene and rehydrated in alcohol and water according to standard procedures. In order to obtain a strong and relevant cyclin E immunohistochemistry staining, slides were microwave treated $\left(96^{\circ} \mathrm{C}\right)$ for a total of $15 \mathrm{~min}(5 \mathrm{~min} \times 3)$ in a buffer containing EDTA $(0.372 \mathrm{~g} / \mathrm{l})$ at $\mathrm{pH}$ 8.0. Several other microwave procedures were tested but the above described procedure was found to be optimal. Microwave treated slides were then placed in a automatic immunohistochemistry staining machine (Ventana 320-202, Ventana Inc., USA) and incubations using HE12 diluted 1:100 and AEC detection as well as hematoxylin-eosin counterstain were performed according to the Ventana program. Similiar staining protocol was applied for $\mathrm{pRB}$ using a polyclonal antibody that recognises both hypo- and hyperphosphorylated form of pRB (C15, Santa Cruz Inc.). Ki-67 stainings were performed manually using MIB-1 antibodies, 1:50 (Immunotech, France) and ABC kits (Vector Laboratories Inc., USA) after a total of 15 min microwave treatment in citrate buffer, pH 6.0.

\subsection{Microscopic evaluation}

Stained sections were microscopically examined and the staining intensity of cyclin E as well as the percentage positive cells determined. The staining intensity of the most intensely stained tumour cells was subjectively estimated using a 3-graded scale where grade $3+$ represented the strongest cyclin $\mathrm{E}$ staining. All slides were evaluated independently twice, and in case of discrepancy, a third examination was performed followed by a conclusive judgement. The percentage cyclin $\mathrm{E}$ and $\mathrm{Ki}-67$ positive cells were determined by calculating at least 300 tumour cells crossing randomly distributed lines.

\subsection{Western blotting and cyclin E protein determinations}

Handling of tumour specimens, protein extraction and determination and Western blotting has been described perviously [25]. Membranes were probed with HE 12 diluted 1:1500 or mouse anti-actin antibodies (Boehringer-Mannheim GmbH., Germany) diluted 1:5000 for one hr and proteins detected with ECL (enhanced chemiluminescence, Amersham Int., England) after a second incubation with peroxidase conjugated anti-mouse antibodies (Amersham Int.). Actin was used to verify equal protein loading on the gel. Relative cyclin E protein concentrations were determined by dividing the optical densities from tumour specimens by that of a cell line standard included on each gel.

\subsection{Statistical methods}

Wilcoxon matched-pairs Signed-ranks test was used to compare the fraction of Ki-67 and cyclin E positive cells. Kaplan-Meier's method was used in calculating survival curves, and comparison between groups was performed using the log-rank test. Survival was defined as the time elapsed from diagnosis to the appearance of death due to breast cancer. If no event occurred, the patient was censored at the time of latest medical check-up, at the time of occurrence of other malignancy or at the time of death caused by intercurrent disease. Multivariate regression analysis was performed by the Cox proportional hazard model for censored data. 


\section{Results}

\subsection{Cyclin E expression in cell lines}

To determine the cell cycle specific expression of cyclin $\mathrm{E}$ we analysed the breast cancer cell lines MCF-7, T-47-D1, ZR-75-1, and MDA-MB-231 for cyclin E protein expression using flow cytometry and two examples including controls are presented in Fig. 1A. The flow cytometric analysis of cyclin E
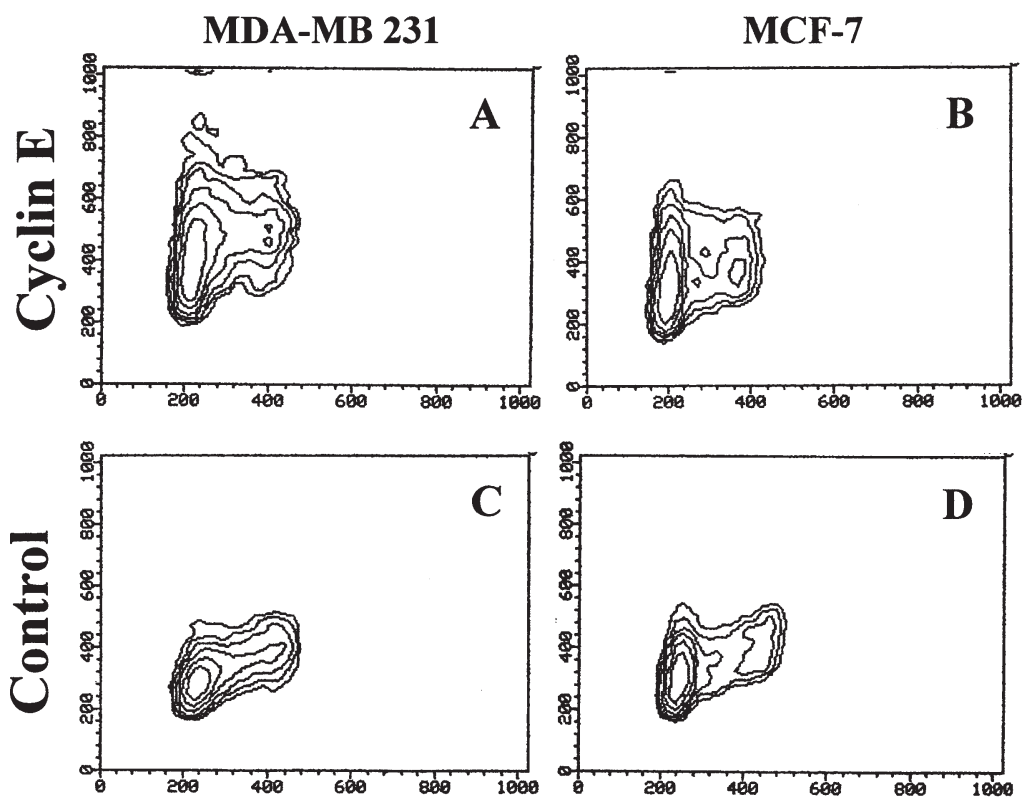

DNA-content

Fig. 1A. Flow cytometric analysis of cyclin E and DNA content (A, B) and control stainings (C, D) using two breast cancer cell lines, MDA-MB 231 and MCF-7.

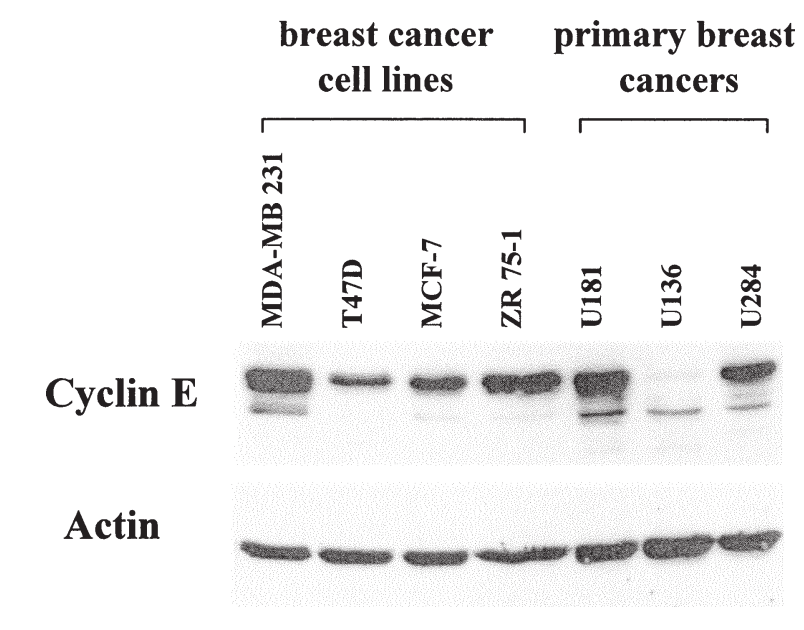

Fig. 1B. Cyclin E and actin Western blotting analysis of breast cancer cell lines MDA-MB 231, T47D, MCF-7, and ZR-75-1 (lane 1-4) and three primary breast cancers U181, U136, and U284 (lane 5-7). 
in relation to DNA content showed for the majority of the cell lines a cell cycle specific distribution of the protein and a fraction of cells with G1-S phase DNA content had the highest cyclin E protein expression. Late $\mathrm{S}$ phase and G2/M cells exhibited lower cyclin $\mathrm{E}$ staining intensities and the signal was almost equal to the control. The cell line T47D produced weaker cyclin E staining with less obvious cell cycle specific distribution of the protein (data not shown). The total amount of cyclin $\mathrm{E}$ was analysed by Western blotting and the results using 4 breast tumour cell lines and three primary breast cancers are presented in Fig. 1B. The breast tumours U181 and U284 expressed high cyclin E levels and have earlier been considered to overexpress cyclin E. The cell lines MDA-MB-231, MCF-7, and ZR-75-1 produced one or two strong cyclin $\mathrm{E}$ bands with intensities equal to the breast tumours U181 and U284 and these findings as well as earlier published results $[15,16]$ support that cyclin $\mathrm{E}$ is highly expressed and presumably overexpressed in some breast cancers and tumour cell lines. The existence of several cyclin $\mathrm{E}$ bands in the Western blots probably represents alternatively spliced forms of cyclin $\mathrm{E}$. The cell cycle specific distribution of cyclin $\mathrm{E}$ in these cell lines with potential overexpression seemed to be conserved as presented in Fig. 1A. This was further confirmed by immunohistochemical analysis of cyclin $\mathrm{E}$, which produced a cell cycle specific staining pattern with several completely negative nuclei using the same cell lines as above (data not shown).

\subsection{Cyclin E expression in breast cancer and relation to proliferation}

We have earlier analysed the relative cyclin E protein level in primary breast cancer specimens using Western blotting [25] and observed that $25 \%$ of the tumours expressed higher and more varied protein levels than the others, proposed to represent tumours with cyclin $\mathrm{E}$ overexpression. To further pursue the significance of cyclin E expression in breast cancer and its relationship to proliferation we performed immunohistochemical analysis using anti-cyclin $\mathrm{E}$ and Ki-67 antibodies in 74 of the tumours earlier studied. Examples of cyclin E immunohistochemical staining of breast tumours are presented in Fig. 2 with indication of the fraction of cyclin E positive cells, staining intensities of cyclin $\mathrm{E}$ and relative cyclin E protein levels determined by Western blotting. The staining patterns of cyclin $\mathrm{E}$ in breast tumours were similar to the patterns obtained with cell lines and we observed distinct nuclear staining with large variations in the percentage and intensities of cyclin E positive tumour cells between tumours. In some tumours a weak cytoplasmic staining was observed which did not interfere with the nuclear staining. Significant correlations were obtained between cyclin E protein levels determined by Western blotting and immunohistochemistry as well as to proliferation as measured by Ki-67 (Table 1). The highest correlation coefficient was obtained between the percentage of cyclin E positive tumour cells and the growth fraction measured by the amount of Ki-67 positive cells $\left(r^{2}=0.64, p<0.0001\right)$ indicating a tight relationship between the periodic expression of cyclin $\mathrm{E}$ and fraction of cells in active cell cycle. We also obtained a significant correlation between the cyclin E protein levels determined by Western blotting and the immunohistochemical determination of cyclin E positive cells, but the $r^{2}$-value increased from 0.40 to 0.49 if the Western blotting results were correlated to the percentage of immunohistochemical cyclin E positive cells multiplied with the intensity suggesting that both the distributions of cyclin $\mathrm{E}$ expression in the cell cycle and the intensity of cyclin E per cell affected the determination of the total amount of cyclin $\mathrm{E}$ by Western blotting. The result of the immunohistochemical analysis of Ki-67 and cyclin $\mathrm{E}$ is shown in Fig. 3 and tumours with a cyclin $\mathrm{E}$ protein level above 0.5 relative to a cell line standard determined by Western blotting, previously used by us to define cyclin E overexpression [25], are indicated. A majority of tumours, including those with potential overexpression of cyclin $\mathrm{E}$, had in general less cyclin E than Ki-67 positive cells $(p<0.0001)$ indicating a conserved cell cycle specific 


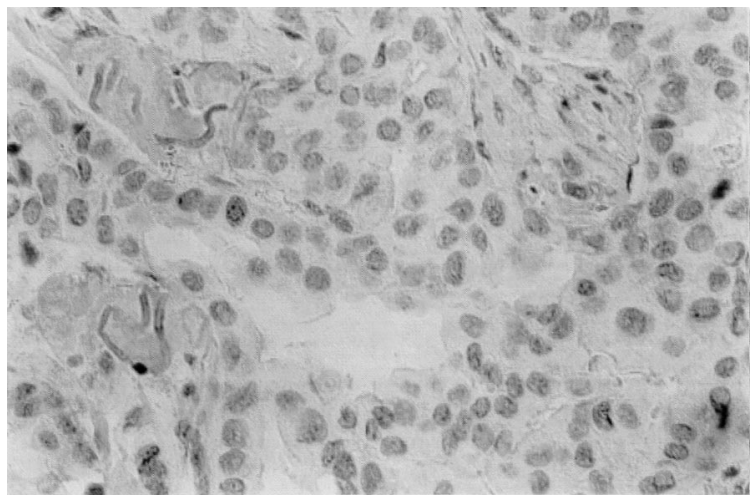

(A)

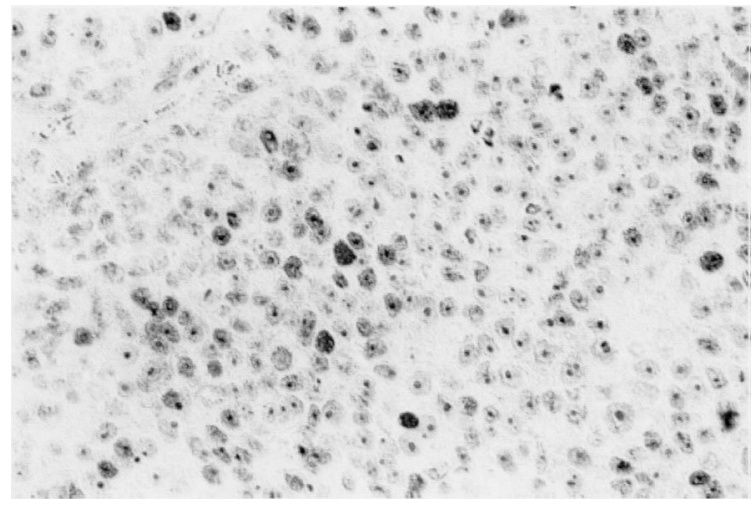

(B)

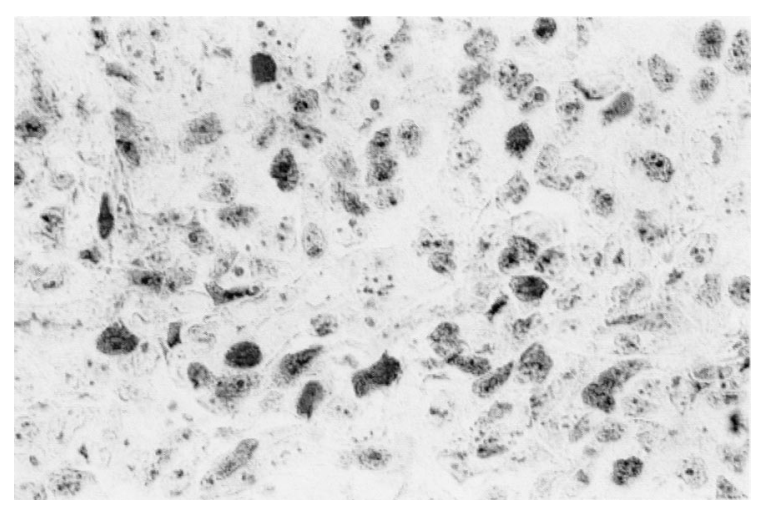

(C)

Fig. 2. Examples of cyclin $E$ immunohistochemical stainings of primary breast cancers. The fraction of cyclin E postive cells were $8 \%$ in (A), $63 \%$ in (B) and $69 \%$ in (C) and the cyclin $E$ intensity of the most intensely stained tumour cells estimated to $2+$ (intermediate) in (A) and 3+ (high) in (B) and (C). The total amount of cyclin E determined by Western blotting was 0.09 in $(\mathrm{A}), 2.43$ in $(\mathrm{B})$ and 4.65 in $(\mathrm{C})$. Tumours $(\mathrm{A})$ and $(\mathrm{C})$ had normal $\mathrm{pRB}$, tumour $(\mathrm{B})$ defect $\mathrm{pRB}$.

expression of cyclin E. Since previous studies have shown a close relationship between pRB inactivation by mutations or increased phosphorylation and cyclin $E$ protein expression $[10,26]$, tumours with defect pRB could be expected to show alterations in the normal periodic expression of cyclin $\mathrm{E}$ in the cell cycle. Our immunohistochemical analysis of cyclin E, however, revealed no apparent differences in the staining patterns between the seven tumours previously considered by us to be pRB defect (lack of nuclear pRB staining) and those considered pRB normal (Fig. 2), implying that pRB independent regulatory molecules in addition to pRB may exist for cyclin $\mathrm{E}$.

In Fig. 4 a summary of the data for cyclin E expression is presented for tumours with respect to various immunohistochemical intensities $(1+$ to $3+)$. Fifty-six out of the 74 tumours were considered to have low/intermediate cyclin E intensities $(1+$ and $2+$ ) while 18 had strong intensities $(3+)$. Minor increase in the percentage of Ki-67 positive cells and cyclin E levels as determined by Western blotting were observed from intensity $1+$ to $2+$ whereas a three and 10 -fold increase, respectively, were observed when advancing to intensity $3+$. This suggests that tumours with strong cyclin E staining had disproportional high cyclin E expression in relation to the proliferative activity, and is further explored in Fig. 5 showing the relationship between percentage of $\mathrm{Ki}-67$ positive cells and cyclin E protein expression as determined 
Table 1

Correlation coefficients ( $r^{2}$-values) for cyclin E protein expression and tumour proliferation as measured by $\mathrm{Ki}-67$ index in breast cancer specimens

\begin{tabular}{|c|c|c|c|c|c|}
\hline & \multicolumn{3}{|c|}{ Cyclin E } & \multirow[t]{2}{*}{ Ki-67 } \\
\hline & & total IH & IH percentage & IH intensity & \\
\hline \multirow{3}{*}{ Cyclin E } & Western blotting & 0.49 & 0.40 & 0.24 & 0.42 \\
\hline & IH percentage & & & 0.51 & 0.64 \\
\hline & IH intensity & & & & 0.33 \\
\hline
\end{tabular}

Cyclin E was quantified using Western blotting and immunohistochemistry (IH). "Total IH" stands for the percentage of cyclin E positive cells multiplied with the intensity. All the presented correlations showed a $p$ value of $<0.0001$.

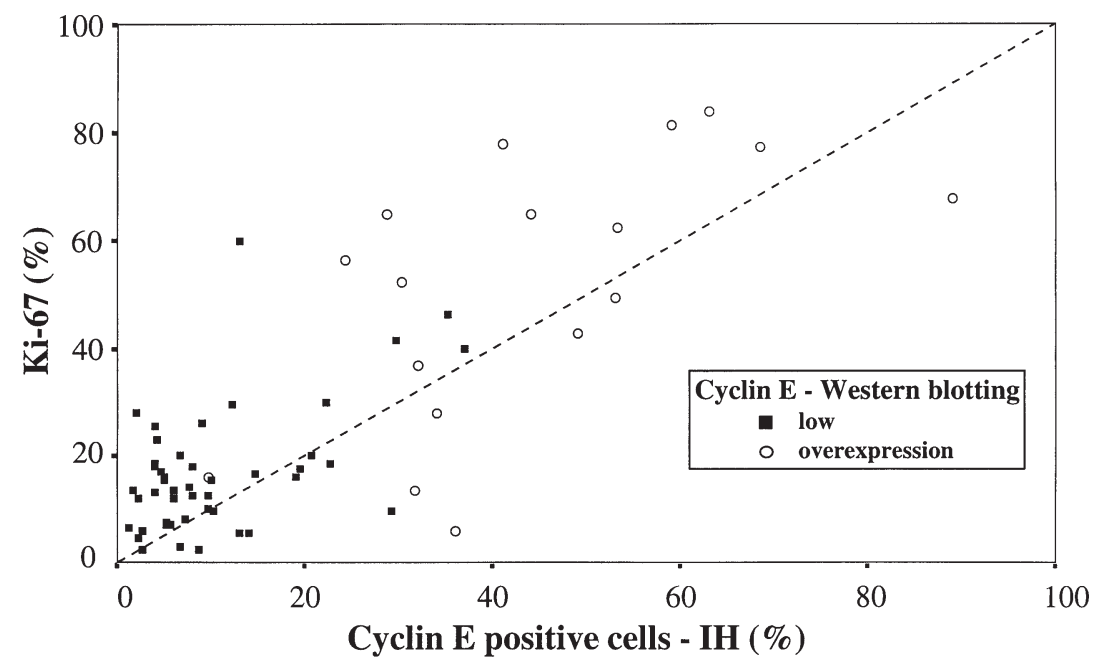

Fig. 3. Scatter plot of the percentage Ki-67 and cyclin E positive cells. Tumours are subdivided into two groups according to the amount of cyclin $\mathrm{E}$ determined by Western blotting.

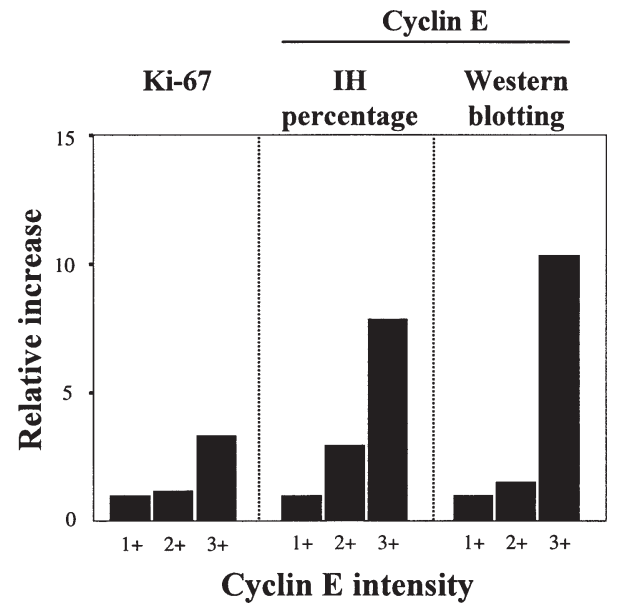

Fig. 4. Mean Ki-67 and cyclin E quantifications according to immunohistochemical staining intensities (1+ to $3+$ ) expressed relative to tumours with $1+$ (low) intensity which was assigned a value of one. IH: immunohistochemistry. 


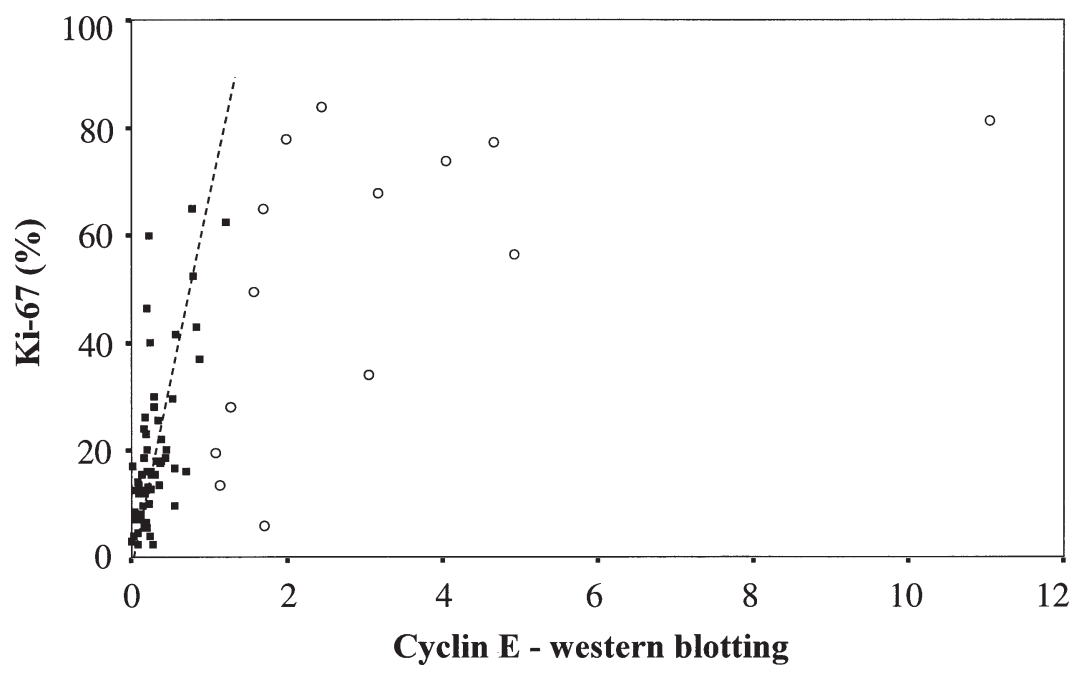

Fig. 5. Scatter plot of the percentage Ki-67 positive cells and total amount of cyclin E determined by Western blotting. Two groups of tumours are defined: one considered following a linear relationship between cyclin E expression and proliferation ( $\mathbf{\square})$, and one considered truly overexpressing cyclin E separated from an exclusively proliferation induced expression of the protein (○). The regression line for the former group is indicated.

by Western blotting. For most tumours, an increase of cyclin E expression was accompanied by a proportional increase in the percentage of Ki-67 positive cells while a group of 14 tumours, defined from the figure, did not adhere to this linear relationship but exhibited higher and more varied cyclin E expressions. These tumours, assuming that cyclin $\mathrm{E}$ is expressed proportionally to the proliferative activity in the normal cell cycle, are suggested to have genuinely deregulated expression of cyclin E detached from the proliferation associated expression that characterised the majority of tumours.

\subsection{Cyclin E expression, proliferation and survival}

In this study we have observed a close relationship between proliferation and the expression of cyclin $\mathrm{E}$ and because the growth fraction is a known risk factor for breast cancer we tried to clarify the importance of cyclin E overexpression in relationship to proliferation. As stated above, potential cyclin $\mathrm{E}$ overexpression in breast tumours cannot be detected by calculating the percentage of cyclin $\mathrm{E}$ positive cells because there seems to be a conserved cell cycle specific expression of cyclin E in the majority of tumours. Another way to evaluate a potential overexpression might be to determine the intensity of the cyclin E staining per cell or to detect high total cyclin E protein levels that are not in relation to the proliferative activity of the tumour. In this study, expression of cyclin E in excess of that expected from proliferation, i.e., the group of tumours in Fig. 5 that did not comply to a linear relationship between total cyclin E protein level as determined by Western blotting and the fraction of Ki-67 positive cells, could be an applicable measurement of cell cycle deregulation due to aberrantly expressed cyclin $\mathrm{E}$. We therefore analysed the survival for patients with stage I-III disease in this group as well as with respect to the various cyclin E quantifications and the proliferative activity as determined by the fraction of Ki-67 positive cells. Significantly impaired prognosis was obtained for patients with tumours of elevated non proliferation associated cyclin E expression ( $p=0.0033$, Fig. 6), high cyclin E levels judged by Western blotting $(p=0.0008)$ and high proliferative activity defined by a Ki-67 index $>20 \%(p=0.0053)$. No impact on the prognosis was observed for cyclin E intensity $(p=0.32)$. In a multivariate regression 


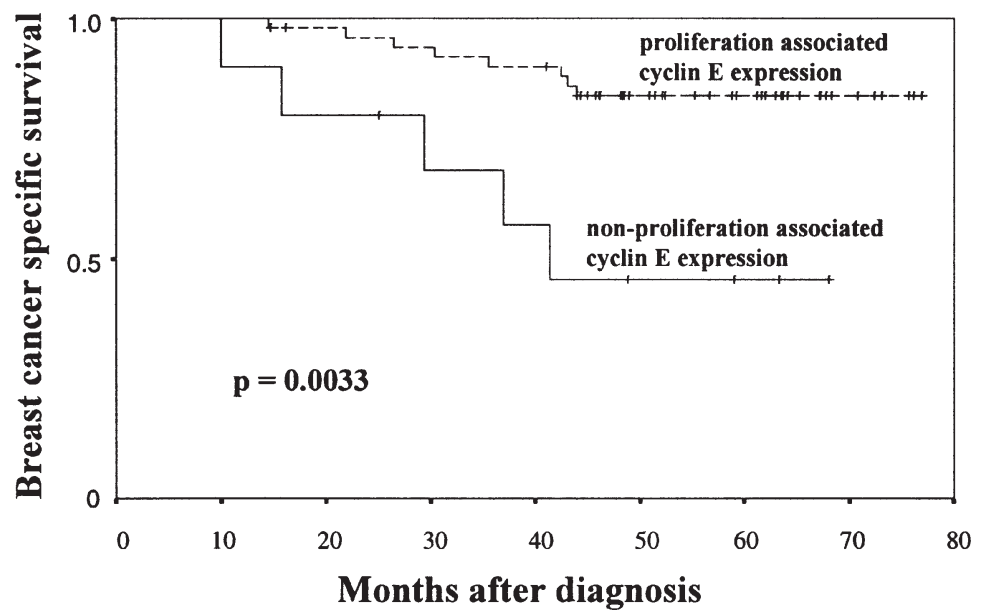

Fig. 6. Kaplan-Meier plots of breast cancer specific survival in 65 patients with stage I-III disease for the two tumour groups defined in Fig. 5.

analysis with covariates showing prognostic significance in the univariate analysis, high cyclin E expression defined by Western blotting were a significant prognostic factor $(p=0.022)$ whereas high fraction of Ki-67 positive cells or elevated non proliferation associated cyclin E expression were not significant factors $(p=0.209$ and $p=0.659)$.

\section{Discussion}

Overexpression of cyclin D1 in breast cancer has been negatively correlated to the proliferative activity $[7,23]$ which contrasts to the proposed function for proteins regulating entrance and passage through the cell cycle thus demanding continued investigations of the relationship between cyclin expression and proliferation in cancer. In this study a significant correlation was obtained between the fraction of cyclin E and Ki-67 positive cells. Similar results have been presented in other immunohistochemical studies using breast and prostate cancer samples [8,22] and data from several flow cytometric studies suggest an association between the expression of cyclin $\mathrm{E}$ and proliferation [11], supporting our data. The fraction of proliferative cells, moreover, correlated to cyclin E intensity which could indicate that recruitment of an increasing number of cells into active cell cycle requires increasing amounts of cyclin E to escape G0. Another possibility comes from the finding that tumours of high cyclin E staining intensity generally did not conform to a linear relationship between total cyclin E protein level and proliferation, arguing that expression of cyclin $\mathrm{E}$ in these, often highly proliferative, tumours might be involved in deregulation of the cell cycle (see below). An additional explanation to the observed correlation between Ki-67 index and cyclin $\mathrm{E}$ intensity could be defective protein turnover of cyclin $\mathrm{E}$ in tumours with high proliferation. Most tumours, however, including those with a high fraction of cyclin E positive cells, had less cyclin $\mathrm{E}$ than Ki-67 positive cells indicating that non proliferating and an additional fraction of actively growing tumour cells were negative for cyclin E protein during part of the cell cycle. Although we cannot discern subtle variations in the level of cell cycle related proteins using immunohistochemistry, and in the present report have not studied the temporal relationship of their expressions to the various cell cycle phases, our findings suggest a conserved cell cycle specific expression of cyclin E in the majority of tumours and rules out a steady protein level throughout the cell cycle. A small fraction of tumours had less Ki-67 
than cyclin E positive cells, albeit still exhibiting distinct variations in the staining intensity of cyclin $\mathrm{E}$ scattered randomly amongst cells, and might represent a group with truly altered cell cycle specificity of cyclin E. Other reports have suggested that breast tumours often suffer disturbance in the normal periodic expression of cyclin E giving rise to a constant level of protein and high associated kinase activity of possible pathogenic importance [15-17]. Some of these reports were based on synchronisation experiments of cell lines including MDA-MB-157 which has known amplification of the cyclin E gene that likely could influence the cell cycle specific expression of cyclin $\mathrm{E}$ and therefore might not be representative for the majority of tumours. In addition, a recent study has found that cyclin E gene amplification is infrequent in breast cancer [6]. Currently we analyse potential cyclin E gene amplification in the tumours presented in this study and these data might clarify the cell cycle specific expression of cyclin $\mathrm{E}$ in tumours with cyclin E gene amplification.

Cyclin E protein is regulated at the transcriptional level and subject to ubiquitin-dependent degradation $[4,10,32]$. Cells with defect pRB and overexpression of cyclin E show noticeable similarities in cell cycle kinetic parameters suggesting that the growth promoting properties conferred by $\mathrm{pRB}$ inactivation could be mediated by induction of cyclin E. In a recent study, however, it was demonstrated that despite loss of periodic transcriptional repression of cyclin E, pRB defective cells still had distinct cell cycle specific variations in cyclin E mRNA level, protein expression and associated CDK2 activity [12]. Several studies on cell lines have identified regulatory molecules in addition to pRB that could be responsible for the cell cycle specific expression of cycle E [4]. Our observation of comparable immunohistochemistry staining of cyclin $\mathrm{E}$ independently of $\mathrm{pRB}$ status in breast tumours therefore supports the existence of a $\mathrm{pRB}$ independent cell cycle specific regulation of cyclin $\mathrm{E}$ in vivo.

Cyclin $\mathrm{E}$ is closely associated with proliferation and possibly with cellular transformation. The fact that tumour cells exhibit various degrees of proliferation makes it difficult to discriminate and exactly define cyclin E's role in these two processes. Certain findings, however, suggest that cyclin E overexpression can be segregated from proliferation and is likely possess a role in tumour development. In a study on diverse malignancies, large alterations in cyclin E expressions between tumour samples and normal adjacent tissue were accompanied by minor variations in the expression of the proliferation marker PCNA [16]. Blasts in the peripheral blood from patients with acute leukemia had uniform low proliferative activity but nevertheless high cyclin E expression suggested to predict the tumour cell's proliferative capacity and disease recurrence [29]. In this study, two different patterns of cyclin E expression were apparent. For most tumours, a close relationship existed between cyclin E expression as determined by Western blotting and Ki-67 index. An equally strong correlation was found between cyclin A expression and the fraction of cells in S, G2 and M phases in a study on lymphomas [27], and we speculate that cyclins in this context serve normal regulatory functions in the cell cycle machinery and that their expressions reliably signal the proliferative activity. In contrast, a minor group of tumours deviated clearly from a linear relationship between cyclin E expression and Ki-67 index all showing higher and considerably more varied cyclin E levels, proposed representing tumours with truly deregulated cell cycle. Tumours with the highest proliferative activity all belonged to this group suggesting that cyclin E overexpression could be a prerequisite for tumour cells to achieve maximal proliferation. This apparent distinction of tumours according to their expression of cyclin $\mathrm{E}$ versus proliferation probably reflects fundamental differences in cell cycle control and argue against a mere proliferation induced expression of cyclin $\mathrm{E}$ in the tumours that overexpressed the protein. Whether this potential mode of cell cycle aberration in breast cancer is a cause or consequence of abnormal expression of cyclin $\mathrm{E}$ is unclear at present. Hence, cyclin $\mathrm{E}$ could be primarily deregulated by structural alterations of its gene or subject to abnormal protein expression or turnover, alternatively, another molecule could be targeted in the neoplastic process, such 
as $\mathrm{pRB}$, and cause overexpression of cyclin $\mathrm{E}$ as well as induction of cell proliferation through either cyclin E dependent or independent pathways. Possible substrates for cyclin E associated kinase activity involved in tumour development has not been fully identified. The transforming ability of cyclin D1 is probably exerted though abnormal phosphorylation and inactivation of $\mathrm{pRB}$ leading to unconstrained G1 progression [21] and a similar mechanism could prevail in tumours with cyclin E overexpression and normal pRB. Activation of the CDK activating phosphatase CDC25A [14] which has proven oncogenic capacity [9] could be another target for cyclin E. Recently, cyclin E's role in tumour development has been more firmly consolidated by the demonstration that cyclin E can cooperate with RAS in the transformation of rat embryonal fibroblasts [24] and the induction of breast tumours in transgenic mice generated to express the protein during during pregnancy and lactation [3], but to address exactly the expression of cyclin $\mathrm{E}$ in relation to proliferation in tumours further characterisation of cyclin $\mathrm{E}$ in the normal and neoplastic cell cycle will be necessary.

Ki-67 index, cyclin E expression determined by Western blotting and elevated non proliferation associated cyclin E expression showed all prognostic significance in this study in contrast to no prognostic importance of immunohistochemically determined cyclin E intensity. Despite the strong correlation between cyclin $\mathrm{E}$ and proliferation, and consequently an overlap in tumours with high cyclin $\mathrm{E}$ expressions and Ki-67 indices, these groups did not identify exactly the same patients. Thus, 6 out of 24 tumours with cyclin E overexpression as determined by Western blotting had less than $20 \% \mathrm{Ki}-67$ positive cells and ten out of 28 tumours with more than $20 \%$ Ki-67 positive cells had low cyclin E levels (Fig. 5). Although cyclin E expression determined by Western blotting was the only significant prognostic factor in a multivariate analysis, delineation and characterisation of groups of tumours with fundamental alterations in cell cycle control, such as tumours with elevated non proliferation associated cyclin E expression could be important in the understanding of basic biological alterations in breast cancer and can probably also prove clinical relevance in certain groups of tumours. Larger studies are nevertheless needed to clarify these issues.

\section{Acknowledgements}

Supported by grants from the Swedish Cancer Society 3813-B96-01XAB and Lion's Cancer Research Foundation in Umeå, Sweden. The authors want to thank Bodil Bäcklund and Inga-Lis Fransson for technical assistance, Ingrid Gustavsson and Katrin Stighall for help with the Ventana and Dr Stefan Emdin for collecting the tumour material.

\section{References}

[1] Y. Akama, W. Yasui, H. Yokozaki, H. Kuniyasu, K. Kitahara, T. Ishikawa and E. Tahara, Frequent amplification of the cyclin E gene in human gastric carcinomas, Jpn. J. Ca. Res. 86 (1995), 617-621.

[2] V. Baldin, J. Lukas, M.J. Marcote, M. Pagano and G. Draetta, Cyclin D1 is a nuclear protein required for cell cycle progression in G1, Genes Dev. 7 (1993), 812-821.

[3] D.M. Bortner and M.P. Rosenberg, Induction of mammary gland hyperplasia and carcinomas in transgenic mice expressing human cyclin E, Mol. Cell Biol. 17 (1997), 453-459.

[4] J. Botz, K. Zerfass-Thome, D. Spitkovsky, H. Delius, B. Vogt, M. Eilers, A. Hatzigeorgiou and P. Jansen-Dürr, Cell cycle regulation of the murine cyclin E gene depends on an E2F binding site in the promotor, Mol. Cell Biol. 16 (1996), 3401-3409.

[5] M.F. Buckley, K.J.E. Sweeney, J.A. Hamilton, R.L. Sini, D.L. Manning, R.I. Nicholson, A. deFazio, C.K.W. Watts, E.A. Musgrove and R.L. Sutherland, Expression and amplification of cyclin genes in human breast cancer, Oncogene 8 (1993), 2127-2133. 
[6] F. Courjal, G. Louason, P. Speiser, D. Katsaros, R. Zeillinger and C. Theillet, Cyclin gene amplification and overexpression in breast and ovarian cancers: evidence for the selection of cyclin D1 in breast and cyclin E in ovarian tumours, Int. J. Cancer 69 (1996), 247-253.

[7] P.J. van Diest, R.J.A.M. Michalides, I. Jannink, P. van der Valk, H.L. Peterse, J.S. de Jong, C.J.L.M. Meijer and J.P.A. Baak, Cyclin D1 expression in invasive breast cancer, Am. J. Pathology 150 (1997), 705-711.

[8] A. Dutta, R. Chandra, L.M. Leiter and S. Lester, Cyclins as markers of tumor proliferation. Immunocytochemical studies in breast cancer, Proc. Natl. Acad. Sci. USA 92 (1995), 5386-5390.

[9] K. Galaktionov, A.K. Lee, J. Eckstein, G. Draetta, J. Meckler, M. Loda and D. Beach, CDC25 phosphatases as potential human oncogenes, Science 269 (1995), 1575-1577.

[10] Y. Geng, E.N. Eaton, M. Picón, J.M. Roberts, A.S. Lundberg, A. Gifford, C. Sardet and R.A. Weinberg, Regulation of cyclin E by E2Fs and retinoblastoma protein, Oncogene 12 (1996), 1173-1180.

[11] J. Gong, F. Traganos and Z. Darzynkiewicz, Growth imbalance and altered expression of cyclins B1, A, E and D3 in MOLT-4 cells synchronized in the cell cycle by inhibitors of DNA replication, Cell Growth Diff. 6 (1995), 1485-1493.

[12] R.E. Herrera, V.P. Sah, B.O. Williams, T.P. Mäkelä, R.A. Weinberg and T. Jacks, Altered cell cycle kinetics, gene expression, and G1 restriction point regulation in Rb-deficient fibroblasts, Mol. Cell Biol. 16 (1996), 2402-2407.

[13] P.W. Hinds, S.F. Dowdy, E.N. Eaton, A. Arnold and R.A. Weinberg, Function of a human cyclin gene as an oncogene, Proc. Natl. Acad. Sci. USA 91 (1994), 709-713.

[14] I. Hoffmann, G. Draetta and E. Karsenti, Activation of the phosphatase activity of human cdc25A by a cdk2-cyclin E dependent phosphorylation at the G1/S transition, EMBO J. 13 (1994), 4302-4310.

[15] K. Keyomarsi and A.B. Pardee, Redundant cyclin overexpression and gene amplification in breast cancer cells, Proc. Natl. Acad. Sci. USA 90 (1993), 1112-1116.

[16] K. Keyomarsi, N. O'Leary, G. Molnar, E. Lees, H.J. Fingert and A.B. Pardee, Cyclin E, a potential prognostic marker for breast cancer, Cancer Res. 54 (1994), 380-385.

[17] K. Keyomarsi, D. Conte, Jr., W. Toyofuku and M.P. Fox, Deregulation of cyclin E in breast cancer, Oncogene 11 (1995), 941-950.

[18] K. Kitahara, W. Yasui, H. Kuniyasu, H. Yokozaki, Y. Akama, S. Yunotani, T. Hisatsugu and E. Tahara, Concurrent amplification of cyclin E and CDK2 genes in colorectal carcinomas, Int. J. Cancer 62 (1995), 25-28.

[19] G.A. Lammie, V. Fantl, R. Smith, E. Schuuring, S. Brookes, R. Michalides, C. Dickson, A. Arnold and G. Peters, D11S287, a putative oncogene on chromosome 11q13, is amplified and expressed in squamous cell and mammary carcinomas and linked to BCL-1, Oncogene 6 (1991), 439-444.

[20] H. Lovec, A. Sewing, F.C. Lucibello, R. Müller and T. Möröy, Oncogenic activity of cyclin D1 revealed through cooperation with Ha-ras: link between cell cycle control and malignant transformation, Oncogene 9 (1994), 323-326.

[21] J. Lukas, L. Aagaard, M. Strauss and J. Bartek, Oncogenic aberrations of $\mathrm{p} 16^{\mathrm{INK} 4 / \mathrm{CDKN} 2}$ and cyclin D1 cooperate to deregulate G1 control, Cancer Res. 55 (1996), 4818-4823.

[22] R.D. Mashal, S. Lester, C. Corless, J.P. Richie, R. Chandra, K.J. Propert and A. Dutta, Expression of cell cycle-regulated proteins in prostate cancer, Cancer Res. 56 (1996), 4159-4163.

[23] R. Michalides, P. Hageman, H. van Tinteren, L. Houben, E. Wientjens, R. Klompmaker and J. Peterse, A clinicopathological study on overexpression of cyclin D1 and p53 in a series of 248 patients with operable breast cancer, Br. J. Cancer $\mathbf{7 3}$ (1996), 728-734.

[24] D. Mumberg, K. Haas, T. Möröy, R. Niedenthal, J.H. Hegemann, M. Funk and R. Müller, Uncoupling of DNA replication and cell cycle progression by human cyclin E, Oncogene 13 (1996), 2493-2497.

[25] N.H. Nielsen, C. Arnerlöv, S.O. Emdin and G. Landberg, Cyclin E overexpression, a negative prognostic factor in breast cancer with strong correlation to oestrogen receptor status, Br. J. Cancer 74 (1996), 874-880.

[26] N.H. Nielsen, S.O. Emdin, J. Cajander, G. Landberg, Deregulation of cyclin E and D1 in breast cancer is associated with inactivation of the retinoblastoma protein, Oncogene 14 (1997), 295-304.

[27] P. Paterlini, A.M. Suberville, F. Zindy, J. Melle, M. Sonnier, J.P. Marie, F. Dreyfus and C. Bréchot, Cyclin A expression in human hematological malignancies: a new marker of cell proliferation, Cancer Res. 53 (1993), 235-238.

[28] D. Resnitzky and S.I. Reed, Different roles for cyclins D1 and E in regulation of the G1-to-S transition, Mol. Cell Biol. 15 (1995), 3463-3469.

[29] R. Scuderi, K.A. Palucka, K. Pokrovskaja, M. Björkholm, K.G. Wiman and P. Pisa, Cyclin E overexpression in relapsed adult acute lymphoblastic leukemias of B-cell lineage, Blood 87 (1996), 3360-3367.

[30] C. Theillet, J. Adnane, P. Szepetowski, M.P. Simon, P. Jeanteur, D. Birnbaum and P. Gaudray, BCL-1 participates in the $11 \mathrm{q} 13$ amplification found in breast cancer, Oncogene 5 (1990), 147-149.

[31] T.C. Wang, R.D. Cardiff, L. Zukerberg, E. Lees, A. Arnold and E.V. Schmidt, Mammary hyperplasia and carcinoma in MMTV-cyclin D1 transgenic mice, Nature 369 (1994), 669-671.

[32] K.A. Won and S.I. Reed, Activation of cyclin E/CDK2 is coupled to site-specific autophosphorylation and ubiquitindependent degradation of cyclin E, EMBO J. 15 (1996), 4182-4193. 


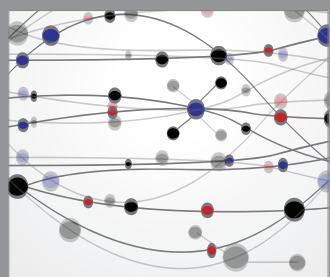

The Scientific World Journal
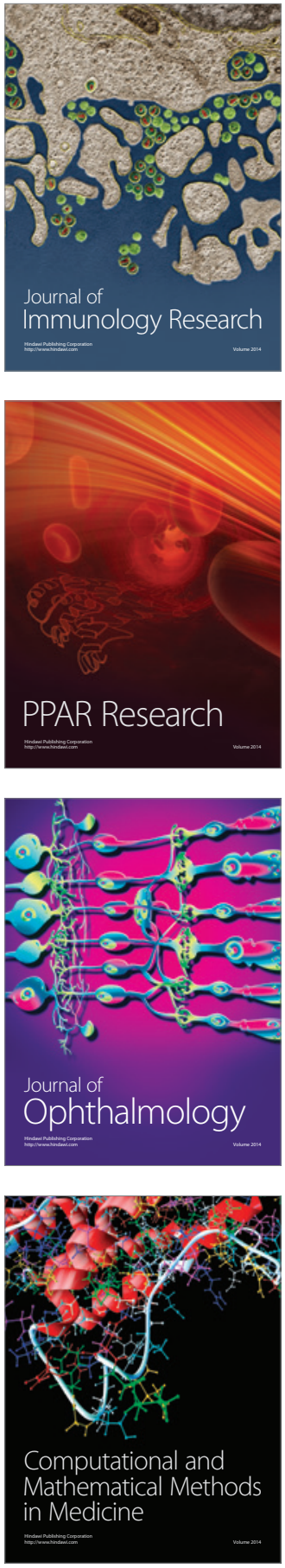

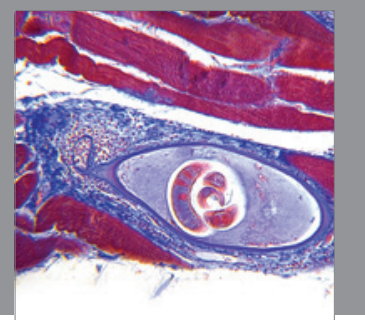

Gastroenterology

Research and Practice
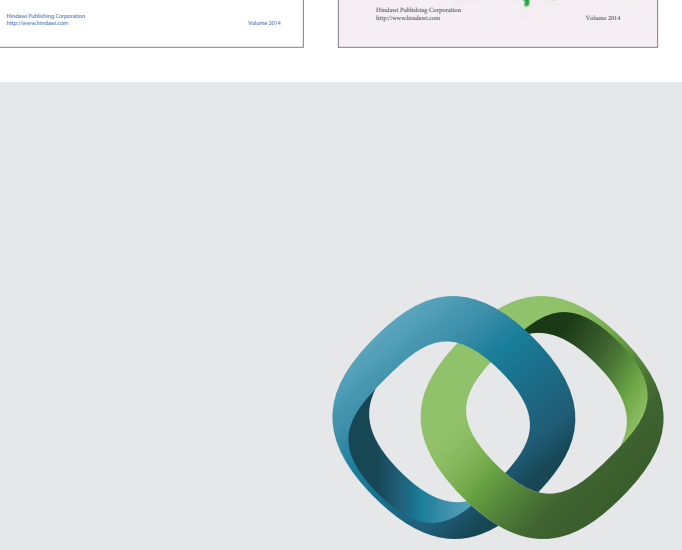

\section{Hindawi}

Submit your manuscripts at

http://www.hindawi.com
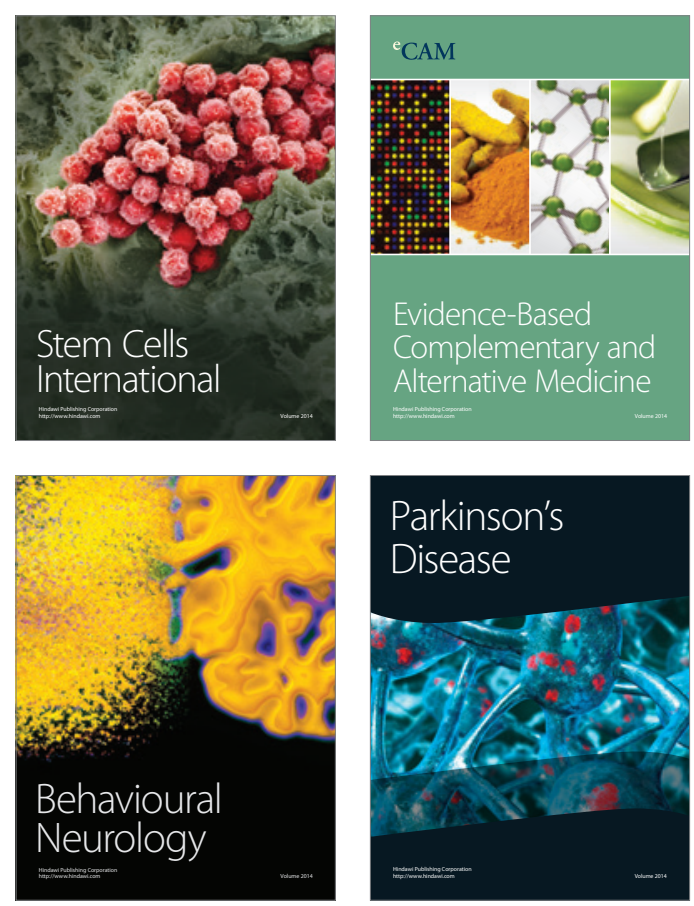

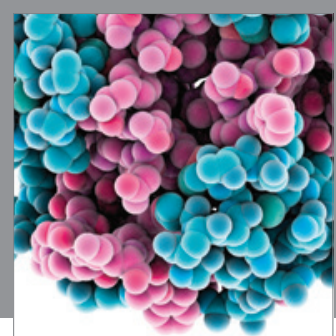

Journal of
Diabetes Research

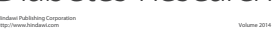

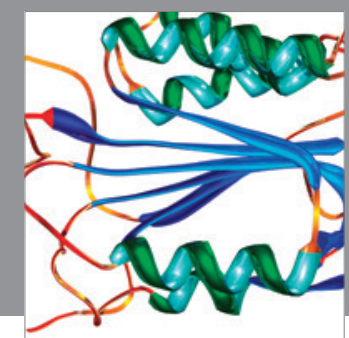

Disease Markers
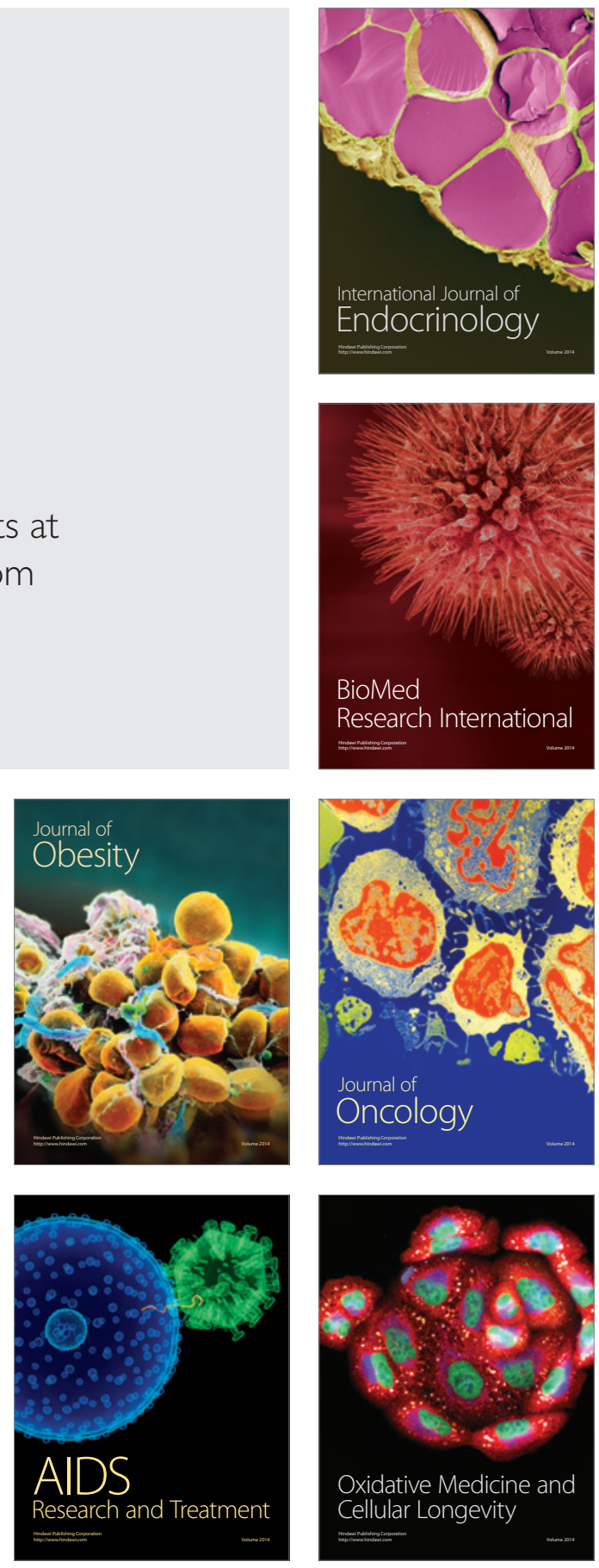